\title{
The Diffuse Near- and Mid-Infrared Emission from the Galaxy
}

\author{
Martin Cohen \\ Radio Astronomy Laboratory, 601 Campbell Hall, University of \\ California, Berkeley, CA 94720, USA
}

\begin{abstract}
Recognition of an isotropic cosmic near-infrared (NIR) and mid-infrared (MIR) background involves the removal of the zodiacal foreground (both scattered and reradiated), of the truly diffuse Galactic foreground (dominated by fluorescent bands of polcyclic aromatic hydrocarbons), and of resolved and unresolved Galactic point sources. I discuss model simulations of the near- and mid-infrared point source sky from which one can assess its particular contribution to the diffuse Galactic infrared foreground. I will also indicate the transitional stage which characterizes our knowledge of fundamental stellar parameters that are essential inputs to any such models. Using the latest version of the SKY model (Wainscoat et al. 1992; Cohen 1993; Cohen 1994; Cohen et al. 1994; Cohen 1995; Ruphy et al. 1997), I will demonstrate matches to deep point source counts for a variety of passbands and galactic latitudes, and will try to quantify the uncertainties achievable in model predictions of the integrated surface brightness due to the smearing of all these foreground point sources.
\end{abstract}

\section{Introduction}

I describe modeling efforts to simulate the Galactic point source foreground in the infrared (IR). After defining the basic issues I will make a brief digression into absolute IR calibration which bears on all "direct" predictions of integrated total surface brightness (TSB) by models.

In the search for Cosmic IR Background Radiation (CIBR), one can finesse the truly diffuse Galactic foreground by careful choice of observing wavelength, as opposed to tackling the unresolved morass of faint stars. The "SKY" model of the point source sky will be discussed in order to demonstrate its proven abilities to match Galactic source counts in a variety of filters, both common (traditional ground-based) and arbitrary (e.g., space-based in the 2-35 $\mu \mathrm{m}$ range). Three distinct approaches have been applied to the prediction of TSB by groups using the SKY model. The advantages and disadvantages of these efforts are discussed. I close by quantifying the probable absolute uncertainties in predicting TSB with SKY and establish its TSB-predictive credentials in an application to mid-IR data. 


\section{The Problem \& the Issues}

The fundamental problem is that measurements of sky brightness contain strong interplanetary and Galactic foregrounds, namely the zodiacal scattered (nearIR) and thermally emitted (mid-IR) radiation, that swamp any CIBR. Indeed, the ultimate limit to the precision with which CIBR can be recognized and quantified is often the uncertainty in the zodiacal modeling (see Ozernoy, this Symposium).

Any direct prediction by models of the light of the many unresolved stars and other point sources that also overlie the CIBR must address the issue of a self-consistent absolute IR calibration. It is crucial that the same absolute calibration applies both to the modeling and to the measurements.

Over the past decade, my colleagues and I have established a common calibration context for IR astronomy (see Cohen et al. 1999 and references therein). This framework unifies ground-based, airborne, and spaceborne photometry and spectroscopy; provides self-consistent, traceable products; has achieved absolute and relative validations from space; has been related to an international reference source; supported NASA's Kuiper Airborne Observatory, and currently supports US DoD airplanes. The calibration of a number of satellites is based on our standards, e.g., COBE/DIRBE, IRTS (the Japanese Infrared Telescope in Space), ISO, MSX, and SIRTF. More than 100 filters are covered including 2MASS and DENIS.

\section{The Diffuse Galactic Foreground}

The spatial structure of the diffuse Galactic foreground is now known, e.g., from MSX images: filaments abound, and are brightest close to the plane. The spectral character of the diffuse Galactic foreground is also known in the NIR and MIR from the Arome experiment (Giard 1989; Ristorcelli et al. 1994) and from the IRTS "NIRS" (1.3-4.0 $\mu \mathrm{m})$ and "MIRS" (4.6-11.6 $\mu \mathrm{m})$ spectrometers (Onaka et al. 1996; Chan et al. 1998; Matsumoto, this Symposium). The PAH bands at $3.3,6.2,7.7,8.7$, and $11.3 \mu \mathrm{m}$ are prominent. At higher Galactic latitudes the same spatio-spectral structures exist but are often too faint to define in the NIR/MIR. Consequently, the ideal location to seek CIBR is, unsurprisingly, at high Galactic latitude where the "cirrus" brightness is minimized and one can seek guidance from the IRAS and DIRBE $100 \mu \mathrm{m}$ maps.

Current knowledge of the wavelengths of the foreground Galactic PAH emission bands and associated features implies that one could successfully bypass these by careful choice of the filters utilized in the search for the CIBR. This would avoid the introduction of further uncertainties by the necessity to remove a PAH band's contribution in some bandpass by scaling with respect to far-IR maps (e.g., for the DIRBE $3.5 \mu \mathrm{m}$ band, Arendt et al. 1998; Wright \& Reese 2000).

Finally, one must attempt to remove the unresolved point source foreground by a physically realistic model, for arbitrary filters, with self-consistent absolute calibration, using the same model from the FUV to MIR because work is carried out on this foreground across the spectrum. 


\section{4. "SKY": Validations of Source Counts}

SKY (Wainscoat et al. 1992; Cohen 1993; Cohen 1994; Cohen et al. 1994; Cohen 1995; Ruphy et al. 1997) is wavelength-flexible because it has an embedded spectral library. Its geometry (disk, bulge, arms, spurs, Gould Belt, molecular ring, and halo) is realistic. It has a rich sky containing 87 different categories of stellar, and nonstellar, point sources. SKY is designed to output differential or cumulative source counts, colors, and integrated TSB, and the 5th version (SKY5) contains the identical absolute calibration framework to that described by Cohen et al. (1999).

\subsection{Star Counts in Traditional Filters}

A variety of validations and tests of SKY have been published. I focus here on two aspects critical to the CIBR: to how faint a magnitude has SKY been tested, and is it truly wavelength-flexible so that it can be meaningfully applied to arbitrary space-based passbands such as those of DIRBE, ISOCAM, ISOPHOT and eventually NGST? To address these issues I will mix more challenging experiments at low Galactic latitudes with validations at high latitudes, where only halo and disk contribute, and which are the most appropriate places to seek CIBR.

Hammersley et al. (1999) have exhaustively tested the 4th version of SKY in hundreds of regions near the Galactic plane in $K$, based upon source counts taken from the Two Micron Galactic Survey by Garzón et al. (1993). Any deviations found between predicted and observed counts have been used to hone the next version of SKY. To provide further constraints on stellar populations, the Tenerife group have also made extensive $V R I$ observations along cuts through the plane at many longitudes. As an example of what can be learned about the combination of stellar populations and extinction, Fig. 1 presents a pair of $V I$ counts at $l=6.8^{\circ}, b=-13.7^{\circ}$, showing the fidelity of SKY to $V \sim 18.5$ and $I \sim 17.5$ in this direction.

The most vital places to validate any point source model that is to be used to remove Galactic foreground are the Galactic poles. At the NGP and SGP only the halo and disk are significant contributors to the observed counts. In such work it is important to sample at least hundreds of square arcmin to minimize Poisson uncertainties. Fig. 2 illustrates $H$-band counts at the NGP by Hammersley (1998), stopping at the approximate magnitude at which galaxies begin to influence the source counts significantly, and using the current standard SKY5 model (i.e., with the Sun displaced $15 \mathrm{pc} \mathrm{N}$ of the plane and with halo:disk normalization 0.5 of that used by SKY1 (Wainscoat et al. 1992)). Using the identical model, we can offer validations to magnitudes as faint as $K=17$ at the SGP (Fig. 3) from the dissertation work of Meadows (1994) at the AAO, using $H-K$ color and source profiles to eliminate contamination of these star counts by external galaxies. Note that the excellent match of predicted and observed counts can be traced to within 1.5 mag of the crossover point between halo and disk. A more recent example, for $1 \mathrm{deg}^{2}$ at the NEP, is the work of Kuemmel \& Wagner (2000), compared with the same SKY5 in Fig. 4. Again there is satisfactory agreement between SKY's predictions and observations to $K \sim 17.5^{m}$. 

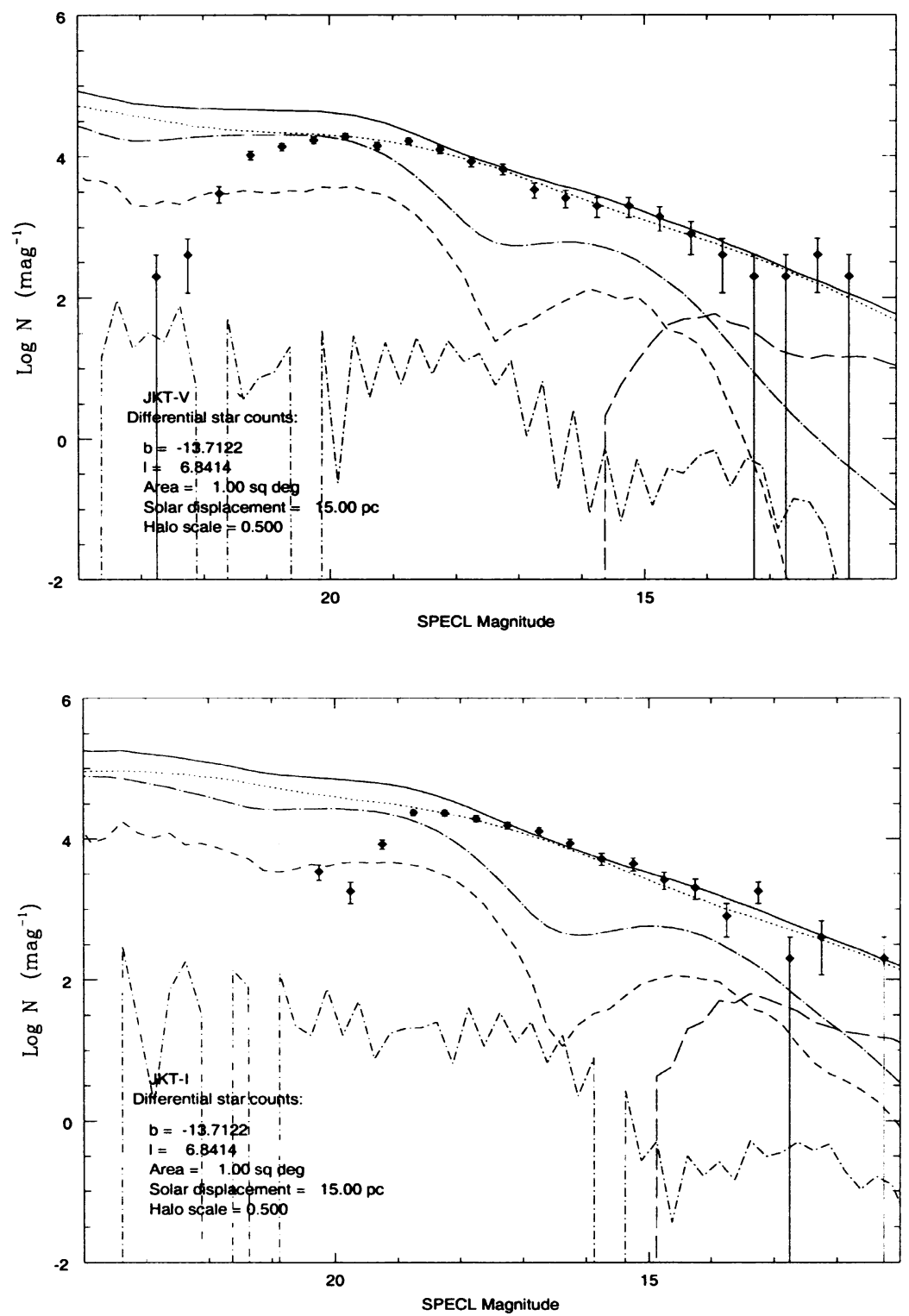

Figure 1. A matched pair of SKY5 predictions in $V$ and $I$ from the Tenerife $V R I$ survey around the Galactic plane. Lines: solid, total predicted counts; dotted, disk; long dashes, spiral arms; short dashdot, molecular ring; short dashes, bulge; long dash-dot, halo 


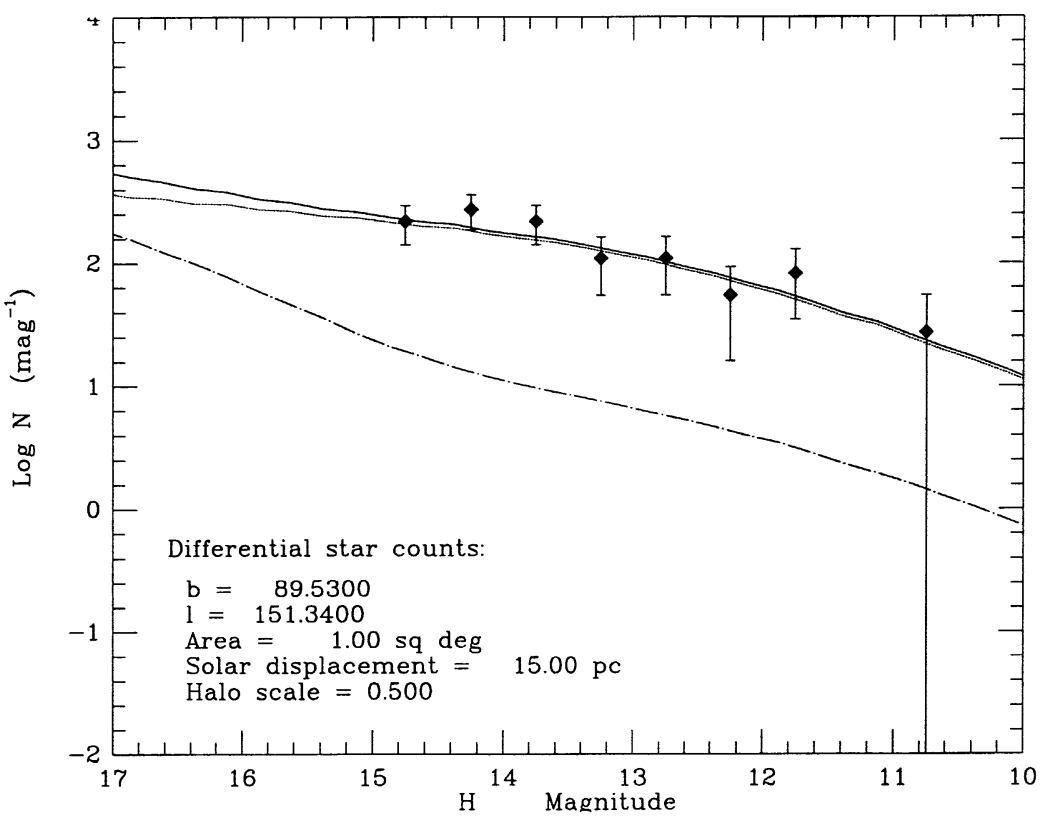

Figure 2. $\quad H$-band star counts at the NGP by Hammersley (1998). Lines as in Fig. 1

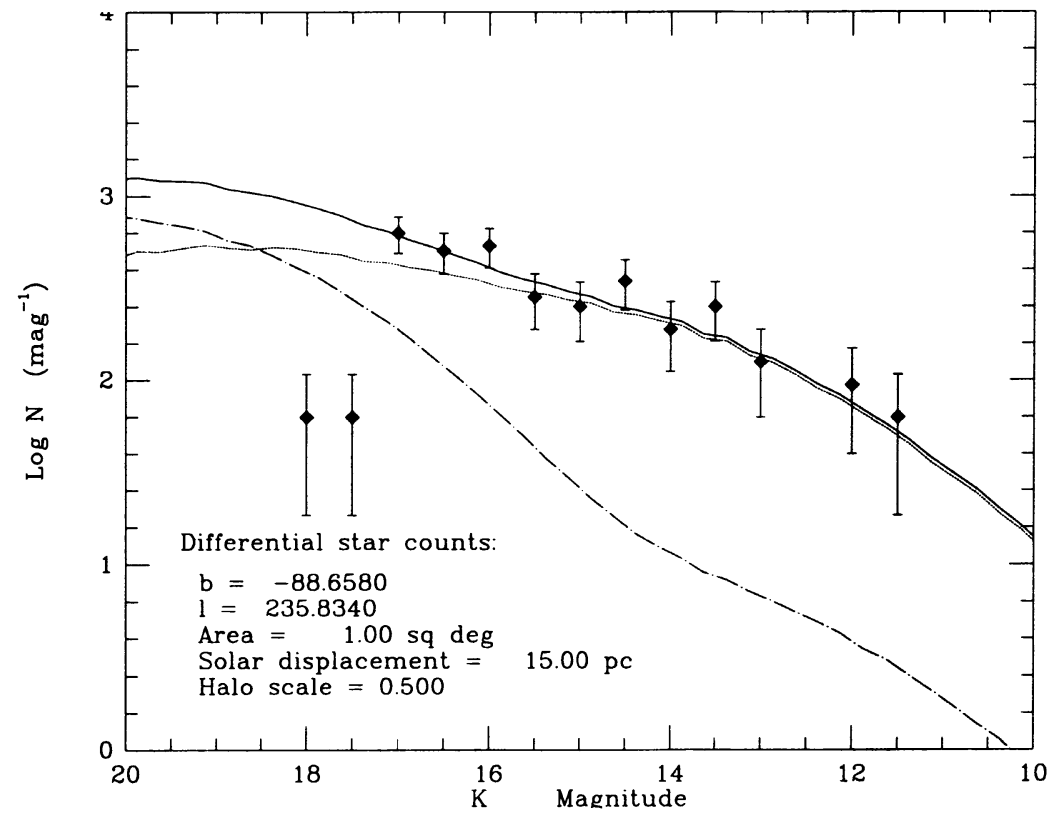

Figure 3. Deep AAO $K$-band star counts at the SGP by Meadows (1994). Lines as in Fig. 1 


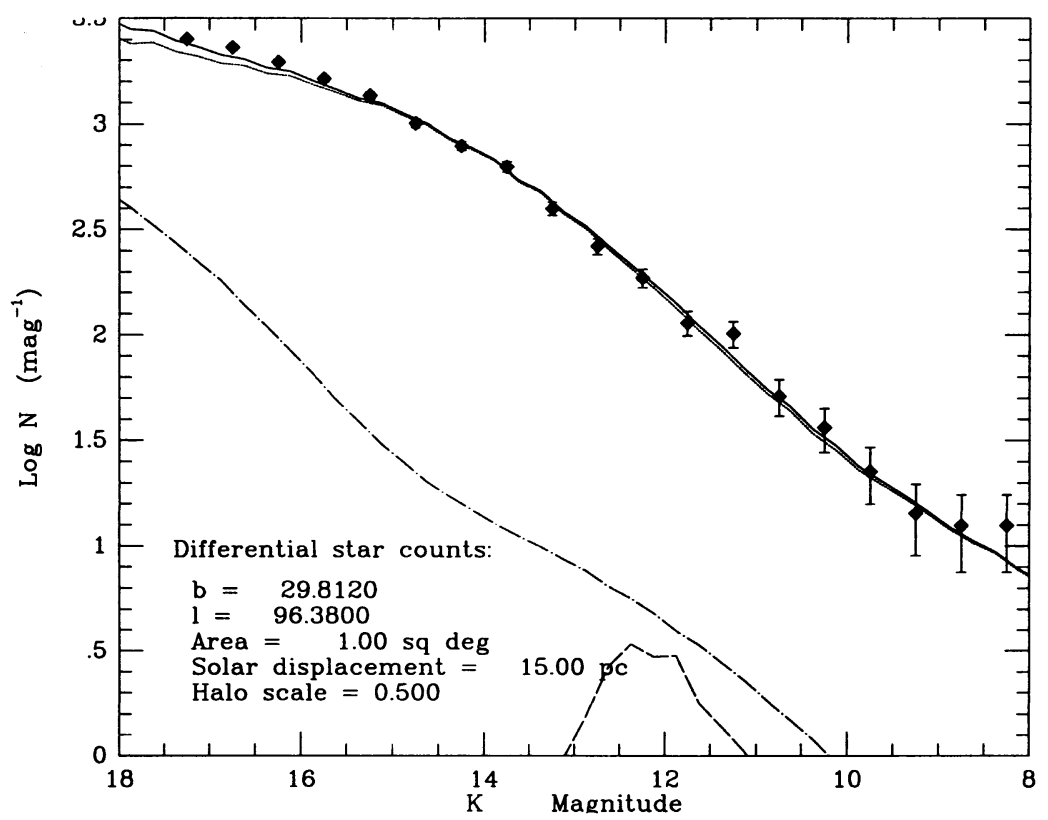

Figure 4. Deep $K$-band star counts at the NEP by Kuemmel \& Wagner (2000). Lines as in Fig. 1

To demonstrate the wavelength flexibility of SKY5 I offer Figs. 5 and 6 , each representing space-based MIR source counts near the Galactic plane. Fig. 5 illustrates the use of SKY5 to test the MSX Point Source catalog version 1.2 (Egan et al. 1999) for processing artifacts and for Malmquist bias in the faintest magnitude bins. Note the contribution from spiral arms at bright magnitudes where the total counts appear to turn up, as predicted. Fig. 6 shows ISOCAM star counts in two fields in or near the plane, comparing SKY5 predictions in two filters: LW7 $(9.6 \mu \mathrm{m})$ and LW3 $(14.3 \mu \mathrm{m})$, from Burgdorf et al. (2000).

\subsection{Application to the Contamination of Galaxy Counts}

Given its fidelity of predicted counts, it is no surprise that a significant industry has grown up around SKY, using it to correct galaxy counts for contamination by faint stars (Saracco et al. 1997; Minezaki et al. 1998; Hall, Green, \& Cohen 1998; Vaisanen et al. (2000) \& this Symposium). Fig. 7 illustrates the usage of SKY5 as a predictor of faint star counts, after validation of its performance on accredited stellar images to the faintest $K$ magnitude at which the combined ESO- $\mathrm{K}^{\prime}$ survey is complete in its point source counts.

\section{TSB Predictions Using SKY}

Given SKY5's proven capability to predict accurate source counts it is no surprise that it has been applied to the prediction of the TSB associated with such counts. Wright \& Reese (2000), Gorjian, Wright, \& Chary (2000), and Arendt et al. 


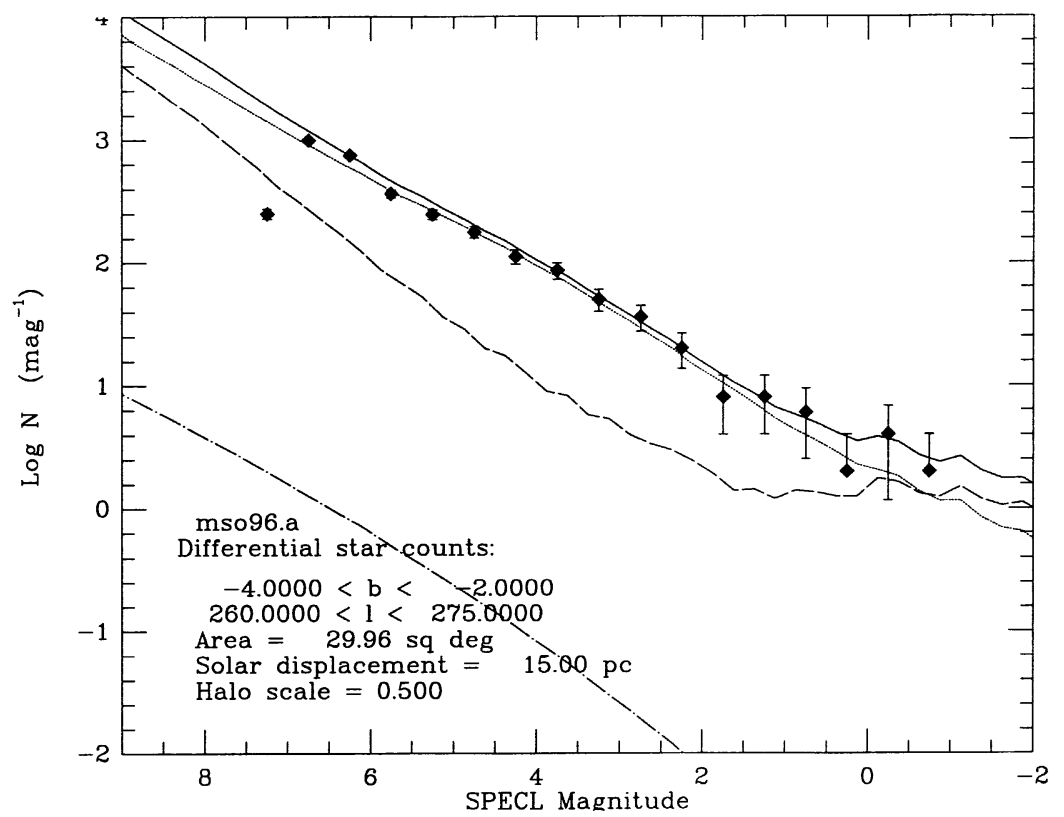

Figure 5. Off-plane star counts from the MSX 8.3- $\mu \mathrm{m}$ band. Lines as in Fig. 1
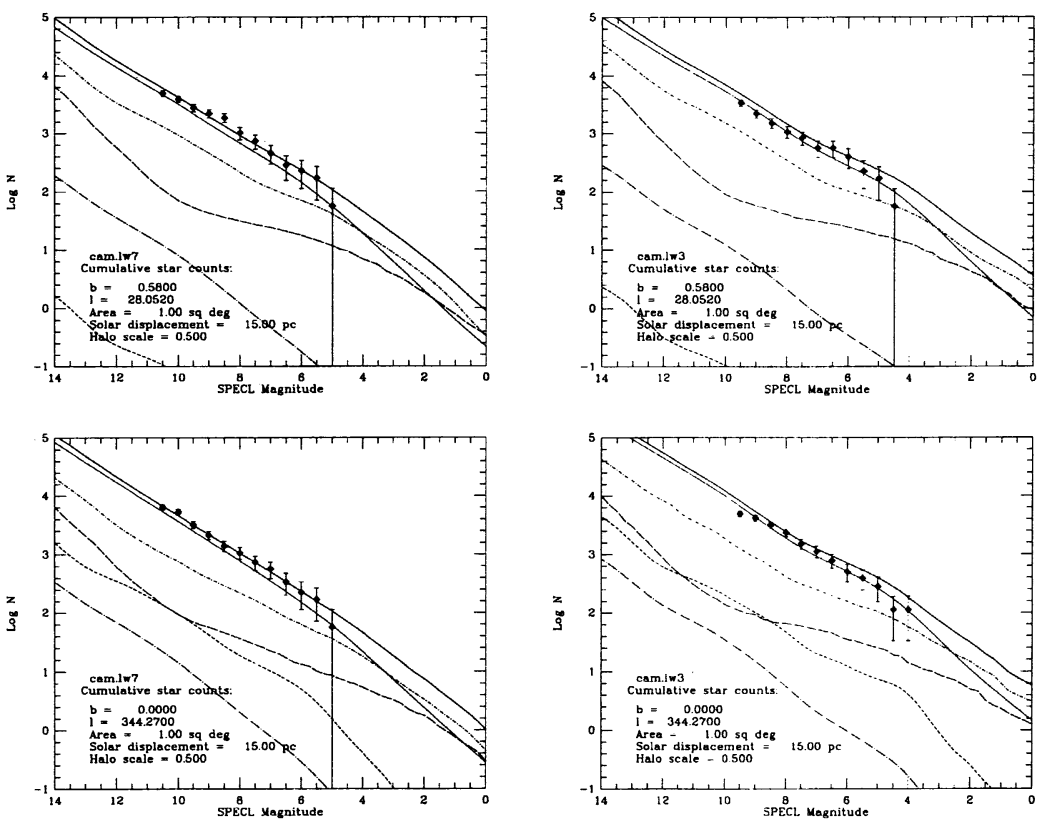

Figure 6. Star counts in two ISOCAM fields in the plane modeled with SKY5 for CAM LW7 and LW3 filters. Lines as in Fig. 1 


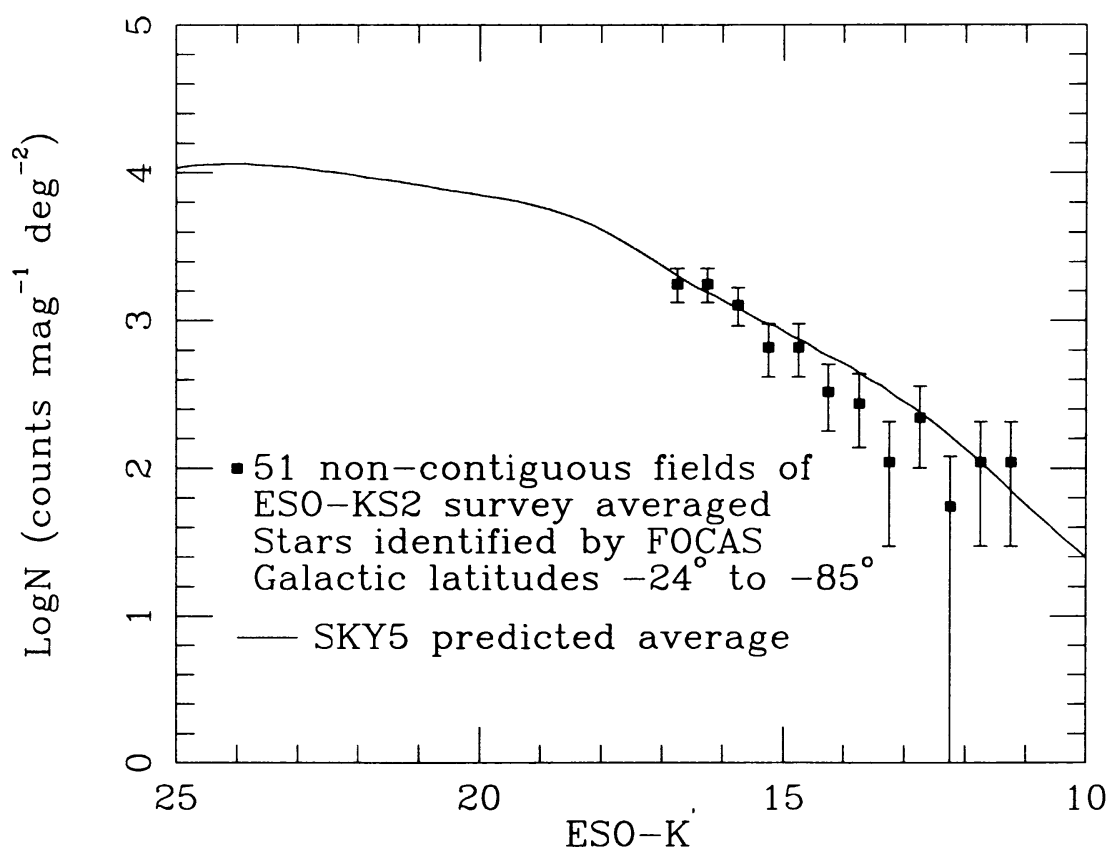

Figure 7. Matched and extrapolated faint star counts in the ESO-K' survey using SKY5

(1998) all used SKY1 to simulate unresolved faint point sources. Matsumoto (this Symposium) also describes the application of the model to this problem but, in this case, used SKY5. There is good reason why one should use SKY5, the current version, as opposed to the dated SKY1. Our knowledge of fundamental stellar parameters such as absolute magnitudes and space densities is now in transition, as we digest the legacy of Hipparcos. SKY5 incorporates this new information. One can quantify the degree of improvement implicit in SKY5. Wright \& Reese (2000) chose to test their own "SKY" against the average of counts from six high and intermediate latitude 2MASS fields. Fig. 8 readily shows the $11 \%$ overprediction of star counts for $6<K<12$ that these authors were obliged to correct for. But SKY5 shows no such bias (the formal match is $1 \pm 3 \%$ for the identical counts and magnitude range) anywhere in the extended range $K=4-15$.

Of course, in all such applications of a model, one never simply adopts the total sky brightness equivalent to simulated counts over the full range of interest. There are so few bright sources that slight fluctuations in real star fields, as compared with modeled counts, lead to substantial differences in TSB because such sources contribute so much to the TSB. One should assess the TSB from the real sources, brighter than the threshold of a survey's $100 \%$ completeness, and extend only to the fainter sources using a model.

Direct comparisons of this nature were made by Arendt et al. (1998) using the difference (DIRBE-minus-SKY1). However, these authors found that their sky was "mottled" by fluctuations after subtraction of SKY1's counts, leading to an extra source of noise that limited their ability to seek the CIBR. Two indirect 


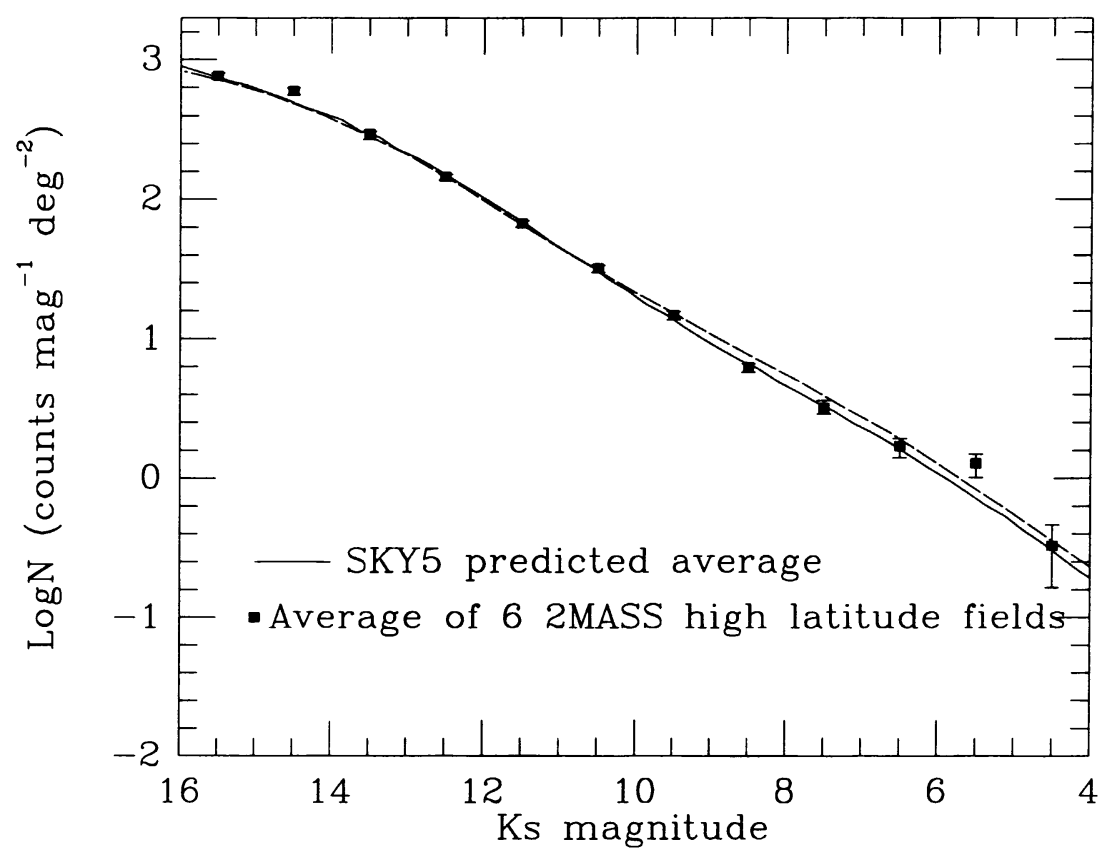

Figure 8. The improvement between SKY5 (solid line) and the variant by Wright \& Reese (2000: long dashed line) for high latitude 2MASS $K$ counts

methods have also been applied. An analysis of such fluctuations is described by Matsumoto (this Symposium). Wright \& Reese (2000) construct a large number of Monte-Carlo simulations of the histogram of SKY pixel values of TSB and compare the mean histogram with that from DIRBE. By this technique, the offset between observed and simulated histogram peaks provides a detection and estimate of the CIBR.

\section{Quantifying the Uncertainties in Predicted TSB}

To quantify what one might expect from SKY5 in the accuracy of prediction of TSB, I consider a concrete example, namely, the sensitivity of SKY5's predicted TSB in DIRBE Band $3(3.5 \mu \mathrm{m})$ at the NGP for magnitudes $>3.19$ (DIRBE's $100 \%$ completeness level for $3.5 \mu \mathrm{m}$ point sources). The solar displacement above the plane, $\mathrm{z}_{\odot}$, is close to $15 \mathrm{pc}$. If we consider the resulting TSBs at 5 and 25 pc $\mathrm{N}$ of the plane this should generously bracket reality. These values of $\mathrm{z}_{\odot}$ lead to uncertainties $\pm 3.5 \%$ in TSB. Similarly, we probably know the halo:disk population ratio in SKY5 (close to 0.5 of the value in SKY1: Wainscoat et al. 1992). But suppose it to be $2 \times$ larger (TSB $+4.1 \%$ ) or smaller (TSB $-5.9 \%$ ). One might then consider as most critical the space density of the faintest and most numerous stars of the disk and halo - SKY's "MLATEV" stars. If it were twice as large or half as large this would imply changes in TSB of $\pm 3.5 \%$. The radial scale length in models is generally taken to be around $3.5 \mathrm{kpc}$, though 

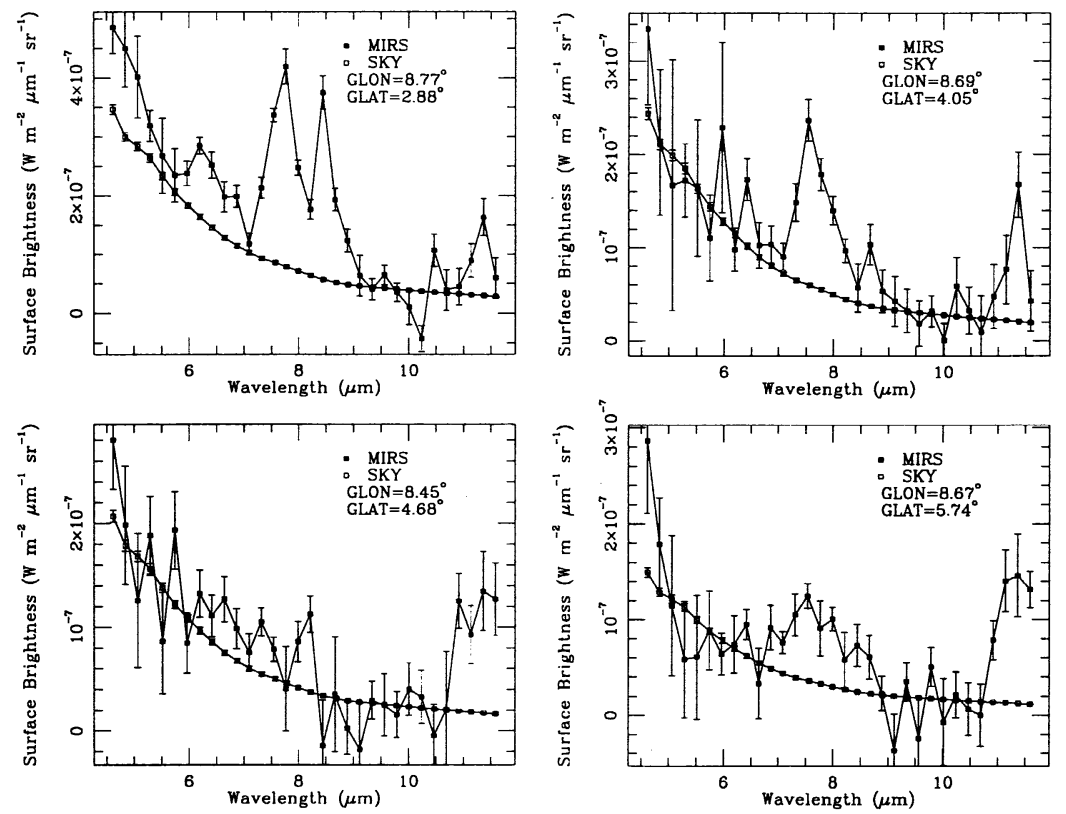

Figure 9. Predicted and observed complete 4.6-11.6 $\mu \mathrm{m}$ IRTS/MIRS spectra of stellar TSB near the Galactic bulge

values as small as $2.5 \mathrm{kpc}$ are often found in analyses of surface brightness maps at $2 \mu \mathrm{m}$, and $4.5 \mathrm{kpc}$ can result from optical TSB maps. The changes in TSB in our test case would then be $-4.0 \%(2.5 \mathrm{kpc})$ and $+2.3 \%(4.5 \mathrm{kpc})$ compared with SKY5's $3.5 \mathrm{kpc}$. Another element that enters the overall error budget is the uncertainty in the absolute conversion between point source magnitudes and physical units, likely of order $2 \%$ for a well-characterized system's relative response curve (detector, filter, all optics, atmosphere if ground-based).

All these error components are independent so addition in quadrature is appropriate, leading to plausible total uncertainties of $\sim 9 \%$.

\section{A Challenging Application to Mid-IR TSB Prediction}

Can one test this expected accuracy? Consider the 4.6-11.6 $\mu \mathrm{m}$ spectra of the sky taken by the IR Telescope in Space Mid-IR Spectrometer (MIRS: Roellig et al. 1994). With its $8^{\prime} \times 8^{\prime}$ aperture and spectral resolution of about 0.3 $\mu \mathrm{m}$, MIRS detected the PAH bands from the foreground cirrus throughout its low latitude coverage of the Galactic plane (Onaka et al. 1996). Chan et al. (1998) present spectra in four low latitude fields in which the requisite removal of zodiacal emission does not pose a significant uncertainty because it is itself overwhelmed by bulge starlight.

Fig. 9 presents their "zodi-subtracted" MIRS data at a longitude of $\sim 8.7^{\circ}$ for four latitudes between about $3^{\circ}$ and $6^{\circ}$. During the past 2 years all 32 separate MIRS passbands have been spectrally recharacterized (the IRTS was 
recovered from orbit by the Shuttle and taken to the ISAS Tokyo laboratories), yielding a final recalibration. The Figure, therefore, offers the newly recalibrated MIRS TSB data (filled squares with measured uncertainties). The open squares likewise represent SKY5's predicted TSB for all 32 MIRS narrow bands in the four spatially distinct locations, using the identical implicit absolute calibration. The comparison is highly encouraging. In spite of the noise in some of the MIRS spectra for regions more distant from the plane, SKY5 clearly indicates that one can provide the underlying continuum emission purely from unresolved starlight. SKY5 deviates nowhere at the $3 \sigma$ level from the observations except within the known PAH spectral features, and is within $10-20 \%$ of the observed continuum TSBs for all spectral channels in the four fields. Possible $(\sim 2 \sigma)$ deviations at the shortest wavelength are likely due to the non-triaxial bulge still inherent in SKY5. (Chan et al. (1998) suggest that a large bulge population of old, low-luminosity, oxygen-rich AGB stars, with high mass-loss rates, contributes the 11-12 $\mu \mathrm{m}$ features in the two highest latitude fields through their circumstellar silicate emission.) The MIRS data are currently unique and it is likely that the comparison of many more of these spectra with SKY5's predictions would prove useful in quantifying what accuracy can really be achieved by a model in estimating TSB.

\section{References}

Arendt, R. et al. 1998, ApJ, 508, 74

Burgdorf, M. et al. 2000, A\&A, in press

Chan, K.-W., Roellig, T. L., Onaka, T., Yamamura, I., \& Tanabe, T. 1998, ApJ, 505, L31

Cohen, M. 1993, AJ, 105, 1860

Cohen, M. 1994, AJ, 107, 582

Cohen, M. 1995 ApJ, 444, 874

Cohen, M., Sasseen, T., \& Bowyer, S. 1994, ApJ, 427, 848

Cohen, M., Walker, R. G., Carter, B., Hammersley, P. L., Kidger, M. R., \& Noguchi, K. 1999, AJ, 117, 1864

Egan., M. P. et al. 1999, The Midcourse Space Experiment Point Source Catalog Version 1.2 Explanatory Guide, Air Force Research Laboratory Technical Report, AFRL-VS-TR 1999-1522

Garzón F., Hammersley P. L., Mahoney T., Calbet X., Selby M. J., Hepburn I., 1993, MNRAS, 264, 773

Giard, M. 1989, A\&A, 215, 92

Gorjian, V., Wright, E. L., \& Chary, R. 2000, ApJ, 536, 550

Hall, P. B., Green, R. F., \& Cohen, M. 1998, ApJS, 119, 1

Hammersley, P. L. 1998, priv. comm.

Hammersley, P. L., Cohen, M., Garzón, F., Mahoney, T., \& Lopez-Corredoira, M. 1999, MNRAS, 308, 333, 1999

Kuemmel, M. W., \& Wagner, S. J. 2000, A\&A, 353, 867

Meadows, V. S. 1994, Ph.D. thesis, Univ. of Sydney, Sydney, N.S.W, Australia 
Minezaki, T., Cohen, M., Kobayashi, Y., Yoshii, Y., \& Peterson, B. A. 1998, AJ, 115,229

Onaka, T., Yamamura, I., Tanabe, T., Roellig, T. L., \& Yuen, L. 1996, PASJ, 48,59

Ristorcelli, I. et al. 1994, A\&A, 286, L23

Roellig, T. L., Onaka, T., McMahon, T. J., \& Tanabe, T. 1994, ApJ, 428, 370

Ruphy, S. R. et al. 1997, A\&A, 326, 597

Saracco, P. et al. 1997, AJ, 114, 887

Vaisanen, P., Tollestrup, E., Willner, S. P., \& Cohen, M. 2000, ApJ, 540, 593

Wainscoat, R. J., Cohen, M., Volk, K., Walker, H. J. \& Schwartz, D. E. 1992, ApJS, 83, 111

Wright, E. L. \& Reese, E. D. 2000, astro-ph/9912523

Discussion

Mike Werner: How much room or need is there for emission from the ISM between the PAH features in your fit to MIRS data?

Martin Cohen: One can quantify this using one of the figures I showed and looking at the wavelength-specific observational uncertainties. But, in general terms, there's no need to appeal to emission in the Galactic foreground in between the several PAH emission bands, at least for the 4.6 to $11.7 \mu \mathrm{m}$ range. At the $3 \sigma$ level, there are no discrepancies that demand dust emission in these spectra (except for the bulge stars postulated by Chan et al. to have strong circumstellar dust shells at $12 \mu \mathrm{m}$ ).

Charley Lineweaver: As the angular resolution of instruments improves, will you need to introduce a parameter to account for more of the total surface brightness being resolved?

Cohen: The philosophy of SKY is to simulate the point source sky. To that extent, it does not address anything that is partially resolved when it calculates integrated surface brightness. Its wavelength range is currently limited to 0.14 to $35 \mu \mathrm{m}$, so it was not designed for applications to the FIR or millimeter range where diffuse radiation overwhelms point sources. 